\title{
Blue phase revival
}

\section{Simulation of cholesteric blue phases using a Landau-de Gennes theory: Effect of an applied electric field}

Jun-ichi Fukuda, Makoto Yoneya, and Hiroshi Yokoyama

Physical Review E 80, 031706 (2009)

Thermodynamics of blue phases in electric fields

O. Henrich, D. Marenduzzo. K. Stratford, and M.E. Cates, Physical Review E 81, 031706 (2010)

\section{Recommended with a Commentary by Slobodan Žumer, University of Ljubljana}

The blue phases (BP) are unique mesophases that occur in a narrow temperature range between the isotropic and the usual cholesteric phases of highly chiral nematogenic materials. In the case of no constraints by external fields or spatial confinement, depending on the strength of chirality and temperature, three thermodynamically distinct blue phases can be found. These fluid phases are characterized solely by spatially modulated orientational order in all 3 dimensions [See for instance Ref.1]. Blue phase III can exist just below the isotropic phase and it exhibits correlated ordering only on short scales; on larger scales it has an amorphous structure with the same symmetry as the isotropic fluid. Its structure has not been definitely resolved yet. The blue phase I and blue phase II exhibit cubic orientational order, corresponding to the space groups $\mathrm{O}_{8}(\mathrm{I} 4132)$ and $\mathrm{O}_{2}(\mathrm{P} 4232)$, respectively, with lattice constants of several hundred nanometers [1,2].

The initial modeling was by Saupe [3], who recognized that a network of $-1 / 2$ disclinations is needed to stabilize highly twisted areas. In the 80's the model was refined [See for instance Refs. 4 and 1] and the current model for BP I\&II, where a network of double twist cylinders and disclination lines form the $\mathrm{O}_{8}$ or $\mathrm{O}_{2}$ cubic lattices, became widely accepted. Rather soon thereafter a semi-analytical approach based on the Landau- de Gennes free energy was used by Hornreich and coworkers to predict the stability diagram of BPs [5]. The special combination of fluidity and three-dimensional crystalline orientational order makes the cubic blue phases highly appealing for photonic applications. Unfortunately until recently the BPs were just interesting systems existing in a 1 degree temperature range between the isotropic and chololesteric phase. This situation was well characterized by a statement of Sir Charles Frank about blue phases in 1983: "They are totally useless, I think, except for one important intellectual use, that providing tangible example of topological oddities, and so helping to bring topology into the public domain of science, from being the private preserve of the few abstract mathematicians and particle theorists." However, recently polymer stabilization was successfully used to widen the temperature stability range of BP [6] to more than $50 \mathrm{~K}$. The polymer network stabilizing the structure on the other side limits the possible tuning of periodicity. Nevertheless, this discovery lead to a possible fast switching system, and thus toward optical shutter applications [7]. More recently a mixture based on bimesogens that lead to BPs stable over a range of up to $50 \mathrm{~K}$, was reported by Coles et al.[8]. The discovery of BPs with wide temperature ranges has led to a revival of interest in this area of research. 
New studies devoted to the phase stability that mostly employ numerical minimization of the Landau de Gennes free energy [9] are proving and extending Hornreich's findings based on the Fourier expansion of the order parameter field [5]. Coles and co-workers examined the effects of flexoelecricity and claimed its crucial role in the stability of their materials [10]. For possible applications, these effects of electric fields are crucial. A preliminary study of electric field effects based on lattice Boltzmann simulations was performed by Alexander et al. [11].

In this comment I focus on two recently published theoretical papers that go much further in detail examining field effects [12 (Paper1),13 (Paper2)]. Both studies use the minimization of the Landaude Gennes free energy expansion in terms of the tensorial order parameter and its derivatives in the one elastic constant approximation. Field effects are included via the dielectric free energy contribution. Both studies address static solutions using conventional finite difference code where adaptation of unite cell size (redshift) is taken into account. Some aspects of dynamics are also taken into account using artificial relaxation kinetics of ordering tensor. The main focus of the Paper 2 is the phase diagram that includes low to intermediate fields while the Paper 1 focus predominantly of electrostriction and in particular to intermediate and strong field effects on structures. Therefore we first examine Paper 2.

Paper 2 [13]: With extensive numerical simulations the authors show how to improve and extend the phase diagram (temperature, chirality, and electric field) obtained by Hornreich and co-workers in a series of papers [See for instance Ref. 5]. Only the case with positive dielectric anisotropy is covered. Particular attention is given to $2 \mathrm{D}$ hexagonal $\mathrm{H}_{2 \mathrm{D}}$ phase and $3 \mathrm{D}$ hexagonal phases $\mathrm{H}_{3 \mathrm{D}}^{\mathrm{a}} \&$ $\mathrm{H}_{3 \mathrm{D}}^{\mathrm{b}}$ that were predicted before for intermediate fields. The calculated phase diagram includes BP I\&II, three hexagonal phases, and isotropic, cholesteric \& nematic phases. The limiting number

(a)

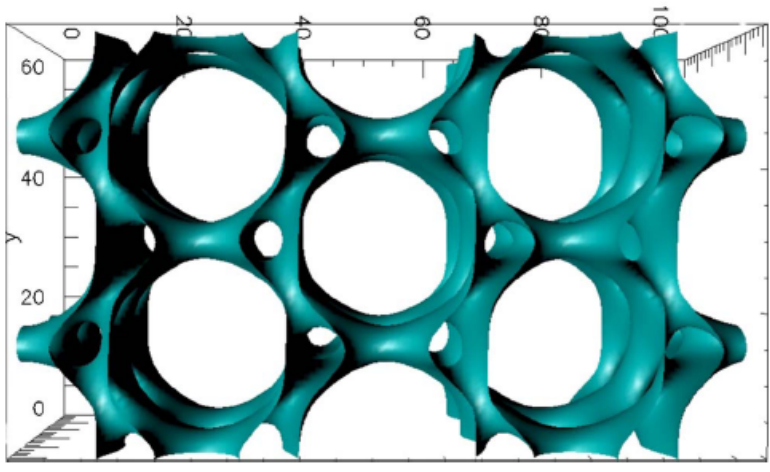

(a)

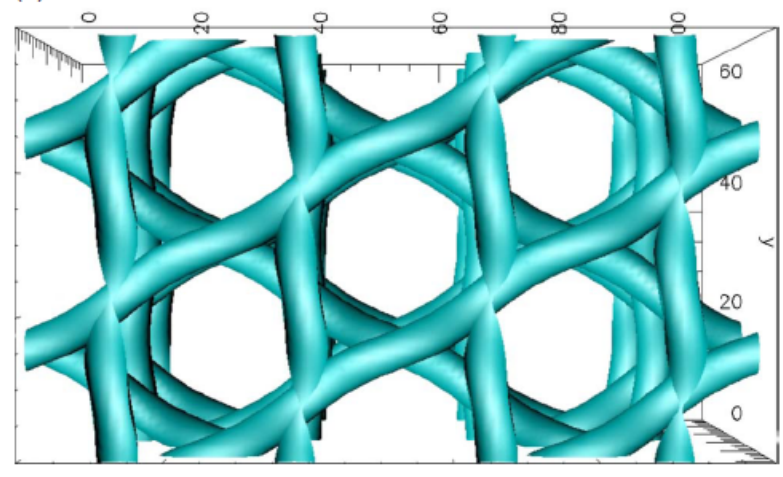

Fig1: Defect structures (surfaces of constant order parameter) for 3D hexagonal phases $\mathrm{H}_{3 \mathrm{D}}^{\mathrm{a}}$ (left) and $\mathrm{H}_{3 \mathrm{D}}^{\mathrm{b}}$ (right) that are stable at intermediate electric fields. Reproduced from Ref 13.

of unit cells covered by simulations prohibit the coverage of the blue phase III. Particular attention is given to the visualization of defect networks by surfaces of constant order parameter, similar to used in nematic colloids [14]. Fig. 1 presents defect structures (surfaces of constant order parameter) of both $\mathrm{H}_{3 \mathrm{D}}$ phases. The hexagonal phases exhibit much larger electrostriction compared to both cubic blue phases.

Paper1[12]: In this paper the authors first focus on electrostriction of BP I\&II structures in fields ranging from weak to very strong values. Materials with positive and negative dielectric anisotropy were considered for field directions (100), (110), and (111). Linear response is found only in low fields (below $\sim 3 \mathrm{~V} / \mu$ ). The second part of the paper is devoted to the restructuring of cubic phases 
after application of a high external field up to $50 \mathrm{~V} / \mu$. In a positive material the final result is a nematic phase while in a negative material the application of a field in the (111) direction leads to a stable hexagonal array of $-1 / 2$ disclinations. The transient phenomena following the application of high fields include a number of interesting disclination network restructurings (See Fig 2!). For instance the split of a $-1 / 2$ disclination into three twist disclinations is observed. More details on the transformation of a twisted line in either a $+1 / 2$ or $-1 / 2$ line observed in numerical simulations of blue phases are discussed in the recent paper by Fukuda [15].
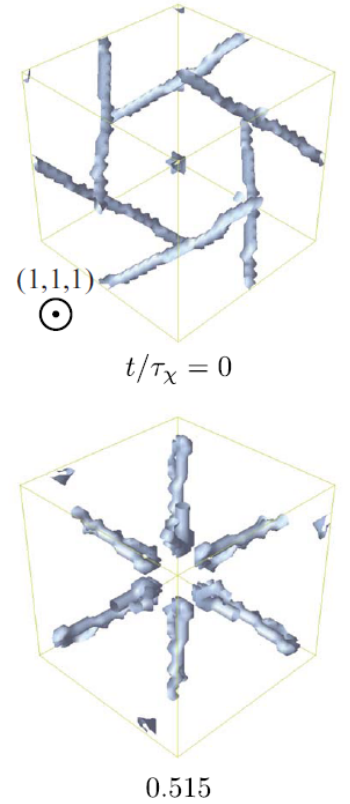
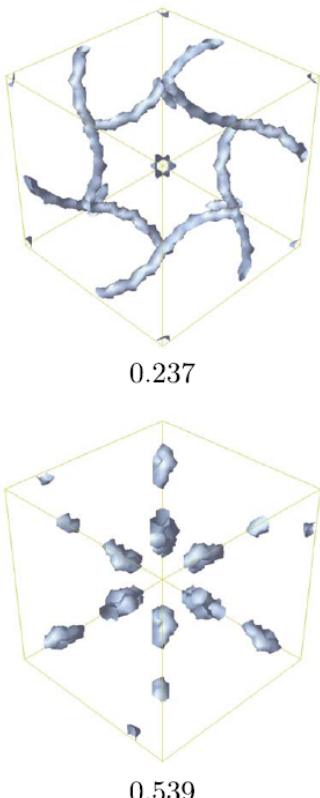

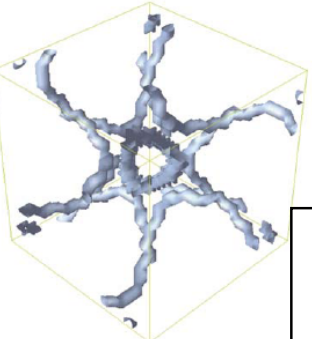

0.476
Fig. 2: Time evolution of the BP II defect structure after the application of the electric field in the diagonal direction of the cubic unit cell. Positive dielectric anisotropy is used. Reproduced from the Ref. 12.

Both studies contribute substantially to the understanding of the behavior of the blue phases in electric fields. This area is particularly important for possible applications of blue phases in light shutters or electrically controlled photonic crystals.

\section{References:}

[1] D. C. Wright and N. D. Mermin, Rev. Mod. Phys. 61, 385 (1989)

[2] P. Oswald and P. Pieranski, Nematic and cholesteric liquid crystals, (Taylor\&Francis, Boca Raton, 2005).

[3] A. Saupe, Mol. Cryst. Liq. Cryst. 7, 59 (1969).

[4] S. Meiboom, J.P. Shetna, P.W. Anderson and W.F. Brinkman Phys. Rev. Lett. 46, 1216 (1981).

[5] R.M. Hornreich, M. Kugler, and Shtrikman, Phys. Rev. Lett. 542099 (1985)

[6] H. Kikuchi, M. Yokota, Y. Hisakado, H. Yang, and T. Kajiyama, Nature Mater. 1, 64 (2002).

[7] Y. Hisakado, H. Kikuchi, T. Nagamura, and T. Kajiyama, Adv. Mater. 17, 96 (2005). Y.

[8] H. J. Coles and M. N. Pivnenko, Nature (London) 436, 997 (2005).

[9] G. P. Alexander and J. M. Yeomans, Phys. Rev. E 74, 061706 (2006).

[10] F. Castles, S. M. Morris, E. M. Terentjev, and H. J. Coles, Phys.Rev. Lett 104, 157801 (2010).

[11] G.P. Alexander and D. Marenduzzo, Europhys. Lett. 81, 66004 (2008).

[12] Jun-ichi Fukuda, Makoto Yoneya, and Hiroshi Yokoyama, Phys.Rev. E 80, 031706 (2009).

[13] O. Henrich, D. Marenduzzo. K. Stratford, and M.E. Cates, Phys.Rev. E 81, 031706 (2010).

[14] I. Musevic, M. Skarabot, U. Tkalec, M. Ravnik, and S. Zumer, Science, 313, 954 (2006).

[15] J. Fukuda, Phys. Rev. E 81, 040701 R (2010). 